Original Contribution

\title{
ANTICIPATION IN THE COGNITIVE STRUCTURE OF SPEECHREADING IN DEAF PUPILS
}

\author{
D. Georgieva*, A. Koleva, G. Valchev
}

Faculty of Education, Trakia University, Stara Zagora, Bulgaria

\begin{abstract}
Anticipation is one of the basic components in the cognitive structure of speechreading. In order to study the probability prediction ability, determining the skills for correct decoding and interpretation of visually perceived oral information by deaf people; an experiment has been carried out involving 91 pupils aged between 7 and 12, integrated into general education schools. Participants in the study have a hearing loss of $\geq 90 \mathrm{~dB}$. The results obtained demonstrate a high degree of correlation between linguistic and meaningful prediction ability and children's speechreading skills. There is also a directly proportional relationship between the chronological age of the students and the level of development of their anticipation ability.
\end{abstract}

Key words: anticipation, speechreading, deafness, cognitive functions, chronological age

\section{INTRODUCTION}

Exploring speechreading is a particularly pressing issue in the contemporary sociopedagogical realities of integrated learning and communication between deaf and hearing students in general education schools. The scientifically verified information on the nature of the relationship between the complex requirements for the processing of linguistic input and the cognitive capacity of nonhearing students is of important scientific and practical significance for the implementation of innovative strategies in the methodology of Audio Speech Therapy and the practice of the school education. Some common characteristics have been outlined in the two theoretical approaches to the character of information processing applied at the level of speech perception, namely the bottom up type - sensory, analytical and the top down type categorical, synthetic, holistic $(1,2)$. The ideas about their interaction lead to the conclusion about the inclusion of context, past experience, and higher cognitive processes in the decoding and overall interpretation of the speech message, regardless of the dominant sensory input. Hence, there has been convincing evidence of deaf children's potential to compensate for deficits in the hearing modality through their visual speech processing abilities (3).

Correspondence to: Diyana Paskaleva Georgieva Faculty of Education, Trakia University - Stara Zagora, Bulgaria, paskaldi1929@abv.bg
Speechreading is a complex process of transcoding the acoustic code into an optical one. The visually segmented acoustic signal itself is limited but there are cognitive schemes that elevate the visual speech perception process beyond the simple passive registration and summation of verbal signals received through the visual sensory channel. Hence, a hypothesis has been put forward that individuals with highly developed visual speech identification skills use higher-order information that is beyond the level of linguistic structures (4).

Anticipation plays a significant role within the context of the cognitive components of speechreading. The stimulus captured by one's vision is matched to a previously created pattern of the expected message, and their overlapping is subjected to a further processing finalized with the decoding of the encoded message.

Anticipation is a phenomenon that attracts the attention of specialists from different fields of science. The term "anticipation" (Latin anticipatio - prediction, preconception, premonition) was introduced for the first time by the German psychologist W. Wundt in 1880 (5). He defines the phenomenon as the ability to present the possible result of an action before the moment of its completion. 
In the past century, the terms "warning activity", "preliminary reflection", "model of the future", "interpolation", "extrapolation", "probability prediction" and "prognostic ability" were coined in relation to the research in animal and human behavioral physiology, cognitive processes and creative activity to indicate the effects of anticipation. $(6,7)$. By summarizing and systematizing the data about anticipation, Lomov and Surkov provided a definition of the term in their fundamental work (8). They describe it as the ability (in the most general sense of the word) to act and accept decisions with a certain temporal and spatial movement in terms of expected future events.

In psycholinguistics, prediction is defined as structural (linguistic) and semantic (9). Semantic prediction is determined by the context and hence by the possible situations, which in turn are related to the use of certain structures, clichés and forms of speech. The semantic level of anticipation is essential in the process of speechreading through visual modality, and in particular with regard to a coherent text. Structural prediction is related to the knowledge of the spectrum of combinations of lexical items. This type of prediction takes place in the process of visual speech recognition at word and phrase level.

In psychology anticipation is interpreted as a universal mechanism of man's psyche. The ability for anticipate is viewed as an immanent characteristic of the human psyche, which is in the position of mutual conditioning between it and the qualities of the different levels of integrated individuality.

The systematic approach to studying anticipation allows the outline of its three main functions: regulatory, cognitive and communicative.

The regulatory function aims at limiting the degree of selection of hypothetical systems corresponding to the spatial and temporal structure of the environment. In accordance with the alleged changes in the circumstances of the external environment, the prediction of the results of actions and the generation of necessary programs are made. This determines the nature and conduct of behavior in the particular situation.

The cognitive function of anticipation is related to the realization of all cognitive processes.

Perception as an ongoing phenomenon always has an element of prediction. Williams postulates that the subject's research activity is governed by prognostic schemes that are plans of the perceptual act (2). As a result of the interaction of these schemes with the actual reality the information obtained modifies the initial prognostic scheme, which in turn further affects the perception.

The mechanisms of anticipation take part in the imagination. The American psychologist U. Neisser supports the idea that images of the imagination, which are in themselves plans of collected information of potentially accessible environment, are a realistic prediction of the future (10).

Anticipation influences attention and mnestic processes. Predicting is an essential component of the memorization, storage and reproduction of information. There is a concept that memorization is not a mechanical record but rather a process that results from the need to select the perceived information. Pre-planning and prognostic sequencing on the part of the subject in the course of his or her verbal behavior are considered essential when selecting information to optimize memorization. When stored in memory, the perceived information is modified and reorganized before being included in the updated mechanism of anticipation in a modified form. At the same time, the retrieval of relevant information at any particular time is determined by the content of the predicted future.

The process of anticipations is directly linked to thinking. In this line of thought we find Brushlinski's concept of thinking as a process of "searching for and discovering what is essentially new" as a form of prediction to be fully relevant (11). Hence, the prognosis of the essentially new is considered as a determinant that gives the direction of searching for the unknown. This is enough to support the view of the unity of thinking and predicting. Researchers share the view that thinking is primarily prediction (12). Thus, anticipation based on the "predicting" brain function is a manifestation of the cognitive activity of the subject.

In fact, predicting is included in the structure of all cognitive levels, and is therefore considered a cognitive psychological process that, like other psychological processes, "penetrates" the entire psyche of a person by being involved in the performance of all kinds of mental activities. 
The communicative function of anticipation is realized in every communicative act, interaction between individuals and the ability to read the emotional state of other people. The process of predicting plays a significant role in understanding someone's speech through the visual sensory channel. For people with hearing impairments where speechreading is an alternative communication system, anticipation is essential for the successful implementation of the process: some events behind the spoken acts as well as the vocabulary describing these events are anticipated, the grammatical forms of the lexical units and the overall syntactic structures are predicted.

In specialized literature, the terms "anticipation" and "probability prediction" are often used interchangeably. At the same time, attempts have been made to differentiate them (13). It is believed that the prediction mechanism is based on the construction of hypotheses regarding the entire content of the text. Anticipation as a component of speech is viewed in a narrow and broad sense. In the narrow sense of the word, this mechanism implies predicting of the elements of words, phrases and sentences. The second meaning of the term "anticipation" coincides with that of the term "probability prediction". Thus, the probability prediction mechanism includes anticipatory skills and is used as a term in a broader sense.

Empirical studies indicate that the visual perception of an oral text is accompanied by some prognostic activity of the receiver, which is combined with his / her personal experience (14). This integrity determines the creation of a complete image, a whole construct of the text. The resulting scheme further governs the perception of the oral information. In this hypothesis-building game which aims at decoding the text, the correct scheme appears. As a result, the content of the text is predicted and the scheme provides a relevant focus of the perceptual process.

It should be noted that these abilities of the individual, such as prediction, anticipation, preliminary synthesis, guessing, are manifested in different types of human activities: mental, verbal, educational and professional. This is a reference to the conclusion that the concept of predicting occupies an important role in a man's life. The ability to predict starts as early as childhood, and can be seen in the child's sensory-motor anticipatory reactions. During the next stages of pre-school age sensory presentations improve and prognostic responses of higher levels of cognitive activity appear. The development of the ability to anticipate at these levels is closely linked to dominant types of activity and the occurrence of new psychic structures. This is the beginning of understanding the probability of events. With the age range being widened the child's experience occupies a more central role in the process of making predictions. At preschool age, the important prerequisites for predicting are generated and they are of paramount importance for the successful realization of the visual speech perception process.

At primary school age, the child is actively involved in learning, acquiring new knowledge, skills and habits. Speech and language are getting more and more detailed. Predicting finds its place within the sentences and partly within the general content of the text. The process of deriving meaning from content prevails over the process of perception. At this stage, the child makes efforts to synthesize not only words within the sentence structure but also demonstrates initial attempts at synthesizing separate phrases within a coherent context. Thus, in normal speech development, the foundations of forming a meaningful guess are laid. The process is particularly important for the development of speechreading skills in deaf children.

At lower secondary school age, probability prediction improves in parallel with students' linguistic, cognitive and life experiences. There is also a sufficiently good level of anticipation. Attention should be paid to the fact that children with normal speech and linguistic development do not need special training concerning the phenomenon. The reason may be found in the natural emergence of the mechanism of anticipation based on intuition (8).

Deaf children with a high level of linguistic competence exhibit an optimal level of anticipation in the process of speech comprehension based on visual modality. Their main foothold is "language sensitivity," the previous speech experience. Familiar and commonly used words can easily be decoded by their limited (in most cases) optical features. At the same time, children with prelinguistic deafness have insufficiently developed probability prediction or their guessing "moves" in the wrong direction. The negative impact of a rich arsenal of effects is manifested in this case: poorly developed lexical and grammatical knowledge, reduced speech experience, limited cognitive 
experience, disturbed auditory control mechanism, etc. Therefore, the development of a reliable anticipatory mechanism, which in turn is a fundamental component of speechreading, requires special training in children with a very severe degree of hearing loss (9).

From what has been said so far, there arises the need for an in-depth study of the anticipation ability in the speechreading process as well as of variations in its development in relation to the chronological age of deaf children integrated in general education schools.

\section{METHODS}

\section{Verbal Stimulus}

The experimental study comprises a verbal stimulus in Bulgarian language - a text. The text is a short story with unfamiliar content, which is consistent with the age and cognitive abilities of the students. It consists of 6 simple grammatical structures, narrative in nature and reflecting processes and phenomena from the surrounding world. By selecting the largest linguistic unit in its role of experimental verbal material, the semantic focus and the orientation towards a specific communicative situation are emphasized.

\section{Design and Procedure}

The linguistic material is selected according to its relevance and is read by the investigator, its access to the auditory modality of the participants in the study being excluded, and vision being used as the only sensory basis. The text is presented twice in a row. The decoding of its content is checked as follows: After presenting the stimulus, the pupils are instructed to draw a picture based on the information received through the visual sensory channel. This is done to check the understanding of the general meaning of the text.

Indicators on which the analysis of the results is based represent a six-point system. Quantitative and verbal assessment is used to establish the level of anticipation. Verbal assessment is formed on the basis of specific indicators. The results of each response are recorded in a separate working protocol for each child.

\section{Equipment and Procedures}

The research has been carried out as a pedagogical experiment. The stimulus has been presented in a relaxed setting and any disturbing factors such as noise, distracting objects, etc. have been eliminated from the communicative situation.
The duration of the entire session varies within 35-40 minutes. The research has been conducted in an environment characterized with a high level of activity and working capacity and a maximum concentration of attention on behalf of the students.

\section{Participants}

91 students have been studied in order to outline specific psychological mechanisms and the mechanism of anticipation in particular, which determines the degree of speechreading skills in specific conditions of deafness. The sample includes participants from 7 to 12 years of age, among which there are 52 boys and 39 girls. According to their hearing status (defined as a hearing loss of $\geq 90 \mathrm{~dB}$ ) and the time of its occurrence, the empirical study differentiates between two groups: students with prelinguistic deafness (44) and students with post-linguistic deafness (47). Regarding the form of school education, all participants in the experiment are integrated into general education schools on the territory of 6 Bulgarian cities.

\section{RESULTS}

The results allow researchers to get a certain idea of the status and features of text-based anticipation. The text itself as a complex communication unit of the highest level is a system of meaningful elements of varying degrees of complexity and significance, functionally combined together by a common concept in a unified semantic structure. At this language level, it is possible to make a more detailed summary, to study and define the characteristics of the mechanisms of probability prediction in the process of speechreading in children and students with hearing impairments.

The frequency distributions of the total number of sentences making up the text which have been visually perceived and properly understood by children demonstrate the level of probability prediction.

The trends in terms of prediction skills in the process of visual perception of the verbal stimulus are as follows: The students, who have visually recognized and illustrated the content of two sentences comprise the highest percentage $(47.25 \%)$. They demonstrate a satisfactory level of development of prognostic ability in this type of speech activity. The visual recognition of two sentences, however, is absolutely insufficient in terms of perceiving the semantic content of the text and understanding the relationship between individual subjects and the actions they 
GEORGIEVA D., et al.

perform. Following are the positive responses of 21 pupils (23.08\%), who have exhibited a good level of successfully coping with the language excerpt. This means that they have correctly predicted half of the semantic content of the text. There are seven instances $(7.69 \%)$ of generally unsatisfactory level of prognostic abilities. 16 students (17.58\%) fall into the category indicating a very good level. They demonstrate the ability to "grasp" the contents of the text by relying on supporting phrases and omitting any superfluous information that is not meaningful or essential for decoding the text. The lowest percentage of respondents $(4.40 \%)$ is a group of students who have received a maximum score of 6 because they have correctly identified and illustrated all the sentences of the text. Thus, they fit within the group with a high level of probability prediction skills. This means that they are capable of drawing logical connection between the sentences and the individual parts of the text on the basis of a well-developed ability to act and make different decisions with specific spatial-temporal movements in terms of future expected events. It confirms the idea that anticipation as a cognitive component, optimizing the speechreading process, is, to a greatest extent, stimulated and improved under the influence of adequate strategies, not the habit of visual perception of speech movements, the development of which is limited by the incomplete and insufficient differentiation of the optical oral code.

Age as a source of variation for the development of the anticipation ability in the speechreading process

One of the tasks formulated in this experiment is to determine the dependence of the level of prognostic ability on chronological age.

On the basis of the conducted literature review, the following hypothesis is drawn: with an increase in chronological age, the probability prediction ability increases.

This study focuses on an age range of 7 to 12 years. There are three groups: pre-school age children (7 years old), primary school age (810 years old) and lower secondary school age (11-12 years).

The data allows the following regularities to be outlined. The two groups of 8-10 year olds $(61,11)$ and 11-12 year olds $(61,77)$ form a homogeneous subgroup due to close means. The mean of the 7-year-olds group is 27.34, which indicates a sensitive difference from the other two age groups. The dynamics of the anticipation ability manifestation increases along with the increase in age. There is a directly proportional relationship between probability prediction ability and the age factor.

In order to determine the differences regarding the source of variation - the chronological age, multiple comparisons have been made to allow the defining of age groups with statistically significant differences, determining the result.

Age is defined as a statistically significant factor for probability prediction on the basis of the data obtained $(\mathrm{F} \sim 5,6 ; \mathrm{P}<0.0001)$. The analysis of the results shows that there is a statistically significant difference between preschool children and the other two age groups in terms of children's anticipation ability in the process of speechreading. As a dynamic cognitive feature, anticipation obviously undergoes certain changes that come with age. At pre-school age (7 years) the ability to socially construct the perspective reality is developed - the distribution of goals and roles, the planning of tasks, the joint activity for the maximum realization of expected constructs. Then, there is a significant leap in prognostic ability at primary school age. Early school-age pupils (8-10) are already able to plan: to set goals and tasks, to undertake activities for their completion; to establish the sequence of actions; to set time limits (deadlines). As a whole, the ability to make predictions is considered as one of the indicators of the child's readiness for the challenges of the next age period. The lack of a statistically significant difference between primary school age and lower secondary school age $(\mathrm{P}=1.000)$ demonstrates the fact that as the age increases, the degree of development of the skills at hand increases steadily. However, it should be noted that the period between 11 and 12 years of age is considered to be the most mature period in human life in terms of the development of the sensory level of anticipation. At the same time, a broad range of useful cognitive experience is acquired and pupils of this age tend to interpret a situation in a new way, mainly from the perspective of the future, with the past and the present remaining in the background, and, hence, a locus of time in the future is formed. From the point of view of the future timeline, one's own self-concept, including a cognitive, emotional and evaluative-volitional component is developed. Adolescents aged 11-12 can view their own reality as a concrete example of a potentially possible alternative. The future as a temporal area is perceived as a closer and more tangible construct. More and more self-control mechanisms are included - a result of the perception of the future and the prediction of 
events. In other words, the self and the social reality are in active construction in accordance with the psychological characteristics of the anticipation of the self and the vision, the perspective of the future. These arguments predetermine the dominant results of lower secondary school students regarding their probability prediction ability in relation to the other two groups.

\section{DISCUSSION}

The empirical results of the research on the level of prediction ability in a specific speech activity, such as speechreading, allow us to outline some significant differences in the realization of this ability in the group of pupils with hearing impairments. One of the most influential arguments in favor of this can be found in the individual psychological qualities of the cognitive processes in the group of participants with profound hearing loss, directly affecting the mechanisms of anticipation that determine the process of speechreading. Very few are the participants in the experiment who show a high level of presentation of the "preliminary reflection" of the real course of various circumstances and correspondingly a high success rate when comprehending the optical codes of speech. It is obvious that these students have the full range of prediction abilities in the communicative-speech model of speechreading. Their thinking is characterized by depth, analytic, awareness and perspective potential. They are capable of theorizing and discovering the cause and effect relationships between subjects and phenomena.

To a great extent they rely on a complete analysis of the causal relationships in the study of relevant phenomena and have a high level of concept awareness. They are capable of understanding the plan's aims and demonstrate profound perspective thinking for the continuously emerging speech hypotheses. Thinking in unsuccessful students is characterized by a low level of flexibility, critical and analytic skills. The grasping of insignificant cause and effect relations affects negatively the ability to understand the essence of the linguistic material - the text, to reveal the causes behind the phenomena, and anticipate the consequences. Decreased level of critical thinking does not allow sufficient analysis of generated hypotheses, which reflects on the ability to draw conclusions and make summaries based on single facts.

In the process of speechreading, the most significant direct effects on the level of probability prediction are observed in memory deficits. The mechanism of these effects is explored in detail in the studies by Campbell and Rönnberg $(3,14)$. The authors find that working memory efficiency in performing language and cognitive tasks depends on controlling the processes of selective retrieval of information related only to the immediate stimuli from the long-term memory. Obviously, deafness conditions predispose to weakened control of the processes of extracting only the necessary information from the deep memory structures. Therefore, irrelevant information signals interfere in the regulation of current linguistic behavior, and these signals in turn affect the mental process and lead to certain manifestations of working memory deficit. Specialized literature places a special emphasis on the influence of hearing deprivation on basic linguistic aspects: lexical, grammatical, pragmatic. The mastery of the semantic content of words takes place in the conditions of a quantitative and qualitative lack of linguistic information, which means that individuals suffering from hearing loss are unable to acquire the meaning of words and respectively real life concepts normal for their age are not formed. This reflects the perceptions of visual speech recognition as a result of the interaction between information from the sensor register and certain information derived from past cognitive experience and stored in the long-term memory. The characteristics of that interaction are clearly different in the population of deaf people.

The analysis clearly indicates the influence of the age factor on the probability prediction skills. A directly proportional relationship has been found between the age of children and pupils with hearing impairment and the results demonstrating the level of the development of their anticipation ability to perceive speech through visual modality. Increase in chronological age leads to an increase in the level of proficiency in meaningful and linguistic prediction, and is characterized by a huge difference between pre-school age ( 7 years) and primary school age (8-9-10 years), followed by steady dynamics. These facts are consistent with empirical data obtained in earlier studies by psychologists $(3,4,8)$ and psycholinguists $(5,9)$. Researchers put forward the idea that prediction itself is an ability which develops spontaneously with age and is not formed as a direct product of learning. In fact, training in lexical and grammatical knowledge takes place at a time when the capacity for their use in identifying and determining logical relations in oral expression 
increases in parallel with experience, extension of vocabulary knowledge, development of cognitive processes, and the general educational level of youngsters. At the same time, children with profound congenital hearing impairment, require an intense search for optimal learning technologies to develop their anticipation ability, which occupies a key position in the speechreading communication model.

\section{CONCLUSION}

Probability prediction is an integral part of speech activity. Visual perception of spoken language as a specific type of speech activity is based not only on the visually perceived oral information but also on its semantic decoding, in other words - on mechanisms that provide global efficiency in understanding the speech of the speaker. In this context, what gains specific importance is the performance of predictions which allow a quick experiencebased selection of adequate meaningful decisions in the process of perceiving oral speech through the visual sensory channel. The results of the experiment show that the efficiency of mastering the skills to perceive the optical characteristics of speech depends on the level of formation of the anticipation mechanism.

The question regarding the development of prognostic competence plays a significant role especially when it comes to the fact that the visual perception of oral speech as a receptive speech model can serve as a solid basis for mastering other personal abilities - reading and writing. Various opinions indicate that achievements in one type of verbal behavior are determined by achievements in other verbal systems.

Knowledge of the cognitive components of the architectonics of speechreading would justify the concepts of preparing therapeutic programs in which the formation of prediction skills would be a part of their structure. Raising awareness of the structural mechanisms of speechreading, and the mechanism of anticipation in particular, could be extremely useful for the practice of Audio Speech Therapy specialists in teaching children this type of skill to such an extent as to encourage the use of basic communication strategies for decoding the speech of the communicating partner.

\section{REFERENCES}

1. Johnson, E. K. \& Jusczyk, P., Word segmentation by 8-month-olds: when speech cues count more than statistics. Journal of Memory and Language, 44, pp. 548-567, 2001.

2. Williams, A., The relationship between two visual communication systems: Reading and lipreading. Journal of. Speech and Hearing Research, 25, pp. 500-503, 1982.

3. Campbell, R., Speechreading past, present and future. First international conference on cognitive hearing science for communication, p. 448, 2011.

4. Marslen-Wilson, W. D. \& Warren, P., Levels of perceptual representation and process in lexical access: words, phonemes, and features. Psychological Review, 101, pp. 653-675, 1994.

5. Kern, S., The Culture of Time and Space: with a new prefece, 1880 - 1918, Harvard University Press Cambridge, Massachusetts Londin, England, 2003.

6. Pavlov, I. P., Complete works, Moscow, 1952.

7. Anokhin, P. K., Cybernetics and integrative brain activity. Questions of psychology, №3, 1967.

8. Lomov, B. F., Surkov, E. N., Anticipation in the structure of activity. Moscow: Science, 1980.

9. Gorman, T. P., Teaching reading at the advanced level. In M. Celce-Muria \& L. Mclntosh (Ed.) Teaching English as a Second or Foreign Language. Rowley, MA: Newbury House, pp. 154-161, 1979.

10.Neisser, U., Cognitive Psychology. New York: Appleton-Century-Crofts, 1967.

11.Brushlinsky, A. V., Psychology of thinking and problem training., Moscow: Knowledge, p. 96, 1983.

12.Eysenck, M.W., \& Keane, M.T., Cognitive psychology (5th ed.). Hove, UK: Erlbaum, 2005.

13.Smirnov, I. B., On the issue of the differentiation between the methodological concepts "anticipation" and "prediction". Foreign languages at school. N 1. pp. 3-6, 2006.

14.Rödnnberg, J., What makes a skilled speechreader? In G. Plant \& K-E. Spens (Eds.), Profound deafness and speech communication (pp. 393-416). London: Whurr Publishers Ltd, 1995. 